\title{
¿EXISTE EL BULLYING EN LA UNIVERSIDAD? APROXIMACIÓN A ESTA REALIDAD CON UNA MUESTRA ESPAÑOLA DE ESTUDIANTES DE GRADO
}

\author{
DOES BULLYING EXIST AT UNIVERSITY? \\ APPROACH TO THIS REALITY WITH A SAMPLE \\ OF SPANISH UNDERGRADUATE STUDENTS
}

\begin{abstract}
ב?
\section{Pilar Royo-García ${ }^{a}$, Cristina Laorden-Gutiérrez ${ }^{a^{*}}$, Montserrat Giménez-Hernández ${ }^{a}$ y Cristina Serrano García ${ }^{a}$}

Fechas de recepción y aceptación: 13 de noviembre de 2019, 11 de mayo de 2020

DOI: 10.46583/edetania_2020.57.510

Resumen: Este artículo analiza la incidencia y las características del bullying en una muestra de estudiantes universitarios en población española. La muestra es de 776 universitarios de cuatro titulaciones de grado relacionadas con educación y psicología (Educación Infantil, Educación Primaria, Educación Social y Psicología). Se utilizó el cuestionario EBIP-Q (Ortega-Ruiz, Del Rey y Casas, 2016) para evaluar la implicación del bullying, adaptándolo a un formato on line. También se evaluó la percepción del ambiente social del aula. Se utilizó una metodología cuantitativa. Se analizaron diferencias de porcentajes en la distribución de los roles de implicación en función del curso $(\chi 2)$, diferencias de medias entre los roles en la percepción del ambiente social del aula (Anova) y los porcentajes de las formas de agresión y de victimización. Los resultados apuntan que existe el bullying en el contexto estudiado: el $12,4 \%$ se considera víctima; el $5 \%$, agresores, y el 2,7 \% agresor-victimizado, encontrando mayor porcentaje de bullying en los cursos superiores y más agresores entre los estudiantes de cuarto que de primero. Las formas más frecuentes de victimización son: ser insultado por otro indirectamente, ser excluido o ignorado, ser insultado directamente o ser el blanco de rumores. Las formas más frecuentes de agresión son: insultar a través de terceras personas o directamente, excluir o ignorar y difundir rumores. Las víctimas expresan menos bienestar en el aula que los agresores y los no implicados. Los resultados apuntan la necesidad de seguir

${ }^{a}$ CU Cardenal Cisneros, adscrito a la Universidad de Alcalá.

* Correspondencia: Centro Universitario Cardenal Cisneros. Avenida Jesuitas, 34. (Alcalá de Henares), 28806 Madrid. España.

E-mail: cristina.laorden@cardenalcisneros.es 
avanzando para conocer las posibles dinámicas universitarias que pueden estar favoreciendo este tipo de relaciones.

Palabras clave: acoso, educación superior, agresor, víctima, convivencia.

Abstract: In this paper, we analyze the impact and characteristics of bullying of Spanish University students. We have a sample of 776 students, distributed in the four academic courses of the degree programs in Teaching Training, Social Education and Psychology. The EBIP-Q (Ortega-Ruiz, Del Rey and Casas, 2016) was used to evaluate the instances of bullying, in an on-line adapted format. The perception of the social environment of the classroom was also evaluated. A quantitative methodology was used. We analyzed percentage differences in the distribution of involvement roles according to the course $(\chi 2)$, mean differences between the roles in the perception of the social environment of the classroom (Anova) and the percentages of the forms of aggression and victimization. The results indicate that there are examples of bullying in the University: $12.4 \%$ consider themselves victims; $5 \%$, aggressors and $2.7 \%$ aggressor-victimized, finding a higher percentage of bullying in the upper grades and more aggressors among the 4th grade students when compared with the 1st grade. We did not find differences according to gender in the different roles. The most frequent forms of victimization are: being insulted by another, being excluded or ignored, being insulted directly or being the target of rumors. The most frequent forms of aggression are: insulting through third parties or directly, excluding or ignoring and spreading rumors. Victims express less well-being in the classroom than aggressors and non-involved people. The results point to the need to continue researching in order to know the possible university dynamics that may be favoring this type of relationship.

Keywords: bullying, Higher Education, aggressor, victim, coexistence.

\section{INTRODUCCIÓN}

Cada vez se va disponiendo de más información sobre lo que ocurre en la Universidad en un tema tan relevante como el acoso entre iguales y se van conociendo algunos resultados reveladores, tal y como constatan las investigaciones sobre este tema que indican que hay presencia de bullying entre los estudiantes en la enseñanza superior (Cowie y Myers, 2015; Perry y Blincoe, 2015; Doğruer y Yaratan, 2014; Sinkonen, Puhakka y Meniläinen, 2012; Coleyshaw, 2010). Así, el bullying no es un fenómeno exclusivo de los niveles educativos precedentes, y parece adquirir matices específicos en el ámbito de la educación superior (Prieto, Carrillo y Lucio, 2015; Romero y Plata, 2015), por lo que resulta importante contribuir a aportar datos sobre sus características. Pero la mayoría de estos estudios se han realizado con muestras extran- 
jeras y son muy pocos los trabajos que incluyen muestras españolas, como el de Caravaca, Falcón, Navarro-Zaragoza, Luna Ruiz-Cabello, Rodriges y Luna Maldonado (2016).

Se entiende por bullying o acoso entre iguales una forma de maltrato por abuso de poder de una de las partes (Olweus, 1998), que conlleva conductas hostiles y poco éticas, intencionales, físicas o psicológicas, directas o indirectas, repetidas en el tiempo y que se realizan con el objetivo de provocar daño en la persona o personas hacia la que se dirige (Ortega, del Rey y Casas, 2013; García Peña, Moncada y Quinterio, 2013; Hoyos, Llanos y Valega, 2012; Monks et al., 2009). El bullying puede manifestarse de forma diversa, a través de conductas físicas (agresión física, golpear o empujar, bloquear el paso, ocultar objetos), verbales (amenazar, chantajear, hacer comentarios negativos) o sociales (hacer burla, muecas o evitar el contacto verbal) (Meriläinen, Puhakka y Sinkkonen, 2015). Estos actos son ofensivos y molestos para la persona acosada y se siente indefensa ante tales hechos, ya que existe un evidente desequilibrio de poder con la persona que agrede. Es importante destacar la naturaleza social del bullying, identificando los diferentes roles que intervienen en la conducta (víctima y agresor, pero también observador y participante), ya que permiten entender cómo se mantienen, refuerzan y configuran las diferentes dinámicas en torno al acoso (Salmivalli, 2010).

Existe una importante investigación en torno al estudio del acoso en las primeras etapas escolares, así como aquella centrada en el acoso laboral (Coleyshaw, 2010), y diferentes autores han encontrado que existe una continuidad en el acoso a lo largo de la vida, es decir, que los roles asociados tanto al papel de agresor como de víctima pueden seguir a la persona desde su etapa escolar hasta su vida profesional (Sinkkonen, Puhakka y Miniläinen, 2012).

En los primeros estudios de Olweus (1998) se recogía una tendencia a la disminución en las conductas de acoso en el paso de la adolescencia a la juventud, debido a los cambios cognitivos propios de esta etapa y a la aparición de nuevos contextos relacionales. También hay estudios que constatan una disminución del maltrato físico, con un repunte de conductas violentas en el contexto de la pareja, las pandillas, el acoso sexual y el acoso laboral (Prieto, Carrillo y Lucio, 2015; Peplet, citado en Vásquez de la Hoz et al., 2010), con unas características y complejidades diferentes a las encontradas en niños y adolescentes (Adams y Lawrence, 2011). 
Sin embargo, otros estudios muestran continuidad entre lo que ocurre en el colegio y en la Universidad. Así, se ha encontrado que aproximadamente el $50 \%$ de los jóvenes que habían sido víctimas o acosadores en la etapa escolar son víctimas o acosadores en la Universidad (Pörhöla et al., citado en Cowie y Myers, 2015; Curwen, McNicho y Sharpe, 2011; Chapell et al., 2006). Por otro lado, se ha encontrado que la probabilidad de que la víctima se convirtiera en agresor es alta en estudiantes universitarios, lo que se explica como una forma de reaccionar ante una situación incontrolable y ante la que percibe poco apoyo para resolverla (Vergel, Martínez y Zafra, 2016; Trujillo y Romero-Acosta, 2016).

Por tanto, frente a lo que cabría esperar según los primeros estudios, fundamentalmente por las características propias de la Universidad (como contexto formativo no obligatorio/libre elección) y de los universitarios, a quienes se les atribuye ciertas competencias cognitivas y sociales y una mayor madurez social (García Peña, Moncada y Quintero, 2013), diferentes resultados más recientes han encontrado porcentajes significativos de bullying. Como se ha comentado anteriormente, casi todos ellos incluyen muestras extranjeras. Así, por ejemplo, varias investigaciones sitúan entre un 5 y un $7 \%$ el porcentaje de estudiantes de universidades finlandesas que expresan haber sido acosados bien por compañeros, bien por miembros de la comunidad educativa (Pörhölä, citado en Cowie y Myer, 2015; Sinkkonen, Puhakka y Meriläinen, 2012; Lappalainen, Meriläinen, Puhakka y Sinkkonen, 2011) y entre un 7 y un $11 \%$ en universidades británicas (NUS Student Experience Report, 2008, y Pörhörlä et al., 2016, respectivamente). En un estudio transcultural, Pörhölä, Cvancara, Kaal, Tampere y Torres (2016) encuentran, además, un porcentaje de víctimas del $25 \%$ en universitarios de Argentina y un $2 \%$ de universitarios de Estonia. A su vez, en un estudio realizado en 22 facultades colombianas de Medicina, se identificó entre un 19,68 y un $30 \%$ de prevalencia de acoso en las universidades (Paredes, Sanabria-Ferrand, González-Quevedo y Moreno, 2010). Los datos más altos los encontramos en un estudio realizado con una muestra española, concretamente estudiantes de Medicina de la Universidad de Murcia que llevaban entre 2 y 6 años en la Universidad, en el que el 62,2 \% de los participantes se identificó como víctima de bullying durante los doce meses anteriores (Caravaca et al., 2016). Las diferencias en los porcentajes de estos estudios podrían explicarse por el marco temporal de la investigación, 
la diversidad cultural y el modo en que el bullying es interpretado en cada uno de los países, aunque algunos autores también apuntan dificultades metodológicas como la imprecisión de las medidas (Benites, 2012). En principio, parece que los datos indican una disminución del problema en la Universidad respecto a etapas anteriores (Cowie y Myers, 2014; Hoyos, Romero, Valega y Molinares, 2009), aunque algunos autores apuntan que esto podría explicarse por la ausencia de estudios sobre el tema (Coleyshaw, 2010) y la falta de consenso a la hora de definir qué se entiende por acoso y su forma de evaluarlo (Goldsmid y Howie, 2014).

En cuanto a los diferentes roles, aparte de lo ya mencionado, el $11 \%$ de estudiantes universitarios del estudio de Meriläinen et al. (2015) expresaron haber sido testigos de situación de acoso en sus universidades. Hoyos, Llanos y Valega (2012) encuentran que el $87 \%$ de los estudiantes se identifica como testigo, un $12 \%$ se reconoce como agresor y el 10,6\% como víctima de acoso. En un estudio realizado por Kunttu y Huttunen (2009), el $25 \%$ dijo haber sufrido acoso en la universidad, de los cuales un 5-6 \% bastante o muy frecuentemente. El 1,7 \% expresaba ser acosador.

En la Universidad, el bullying adquiere diferentes formas e incluye conductas como difundir rumores desagradables relacionados con la edad, el sexo, la raza o la discapacidad; ridiculizar o denigrar a alguien; excluir socialmente; conductas sexuales no deseadas, y amenazas tanto directas como on line (Cowie y Myers, 2015). Si comparamos estos tipos de bullying con lo encontrado en estudios nacionales e internacionales en escolares, se observa que todas las formas de maltrato se presentan en el contexto universitario, siendo más frecuentes el maltrato verbal y la violencia psicológica, seguido de la exclusión social y, en menor proporción, el maltrato físico directo e indirecto (Vergel, Martínez y Zafra, 2016; Hoyos, Llanos y Valega, 2012). Los diferentes estudios encuentran que los universitarios no acuden a agresiones físicas en comparación con etapas anteriores.

En cuanto a la frecuencia, Meriläinen et al. (2015) han encontrado que uno de cada siete estudiantes había sufrido acoso a diario y uno de cada tres, varias intimidaciones un par de veces al mes; el $50 \%$ de los casos fue acoso público directo (por ejemplo, discriminación, trato injusto y presión). Atendiendo al lugar, se ha encontrado que entre un 20 y un $30 \%$ de las situaciones 
de acoso se producen en el aula de clase (Vergel, Martínez y Zafra, 2016; Meriläinen, Puhakka y Sinkkonen, 2015).

Con relación al género, parece confirmarse que los hombres son más veces agresores que las mujeres (Pörhöblä, Cvancara, Kaal, Tampere y Torres, 2016; Hoyos, Llanos y Valega, 2012; Nansel et al., 2001), mientras que las mujeres, en estas situaciones, se relacionan con más frecuencia con el rol de testigo (Hoyos, Llanos y Valega, 2012). Atendiendo al género de las víctimas, los resultados no son definitivos. En el estudio transcultural de Pörhölä et al. (2016), solo se encuentran diferencias significativas en víctimas mujeres en Finlandia, siendo la forma más frecuente de bullying en mujeres, en los cuatro países estudiados, la crítica injustificada, el menosprecio o la humillación (donde oscila entre el 13 y el $15 \%$ ). Por el contrario, los varones argentinos informaron con más frecuencia de ataques verbales, como insultos o amenazas, y los varones estadounidenses, de burlas o críticas sobre cualidades personales, como las formas más frecuentes de acoso.

Hoyos, Llanos y Valega (2012) encuentran que las mujeres que se reconocen como agresoras destacan en las modalidades de ignorar (46,7 \%) y hablar mal de otro $(20 \%)$, mientras que los varones ponen apodos $(29,6 \%)$ e insul$\tan (14,8 \%)$ con más frecuencia que las chicas. En este estudio los hombres agresores también reportan que ignoran, pero que lo hacen en una proporción menor que las mujeres, hecho que coincide con otros estudios en universitarios y escolares (Hoyos, Llanos y Valega, 2012).

Para explicar la incidencia del bullying universitario, algunos autores han apelado a la propia estructura universitaria y a las nuevas capacidades que adquiere el estudiante. Por un lado, la forma en que se configura la docencia, las dinámicas del aula, las disciplinas académicas y las políticas universitarias que buscan proteger posibles posiciones de poder, y por otro, la propia capacidad cognitiva de los estudiantes para resaltar las diferencias de sus compañeros, la necesidad de protagonismo o liderazgo, así como las circunstancias vitales propias de esta etapa (terminación de relaciones de pareja, celos, etc.) o las necesidades específicas y/o dificultades de algunos estudiantes, podrían explicar los porcentajes de bullying encontrado en la Universidad (Vergel, Martínez y Zafra, 2016; McDougall, citado en Hoyos, Llanos y Valega, 2012), aunque no cabe duda de que es necesaria una mayor investigación en este tema. 
La relevancia de todos estos resultados destaca no solo por lo sorprendente de encontrar acoso en un contexto con expectativas altas de formación, sino por las consecuencias que se constatan tanto en víctimas como en agresores. Isaacs et al. (2008) encuentran importantes repercusiones negativas en la salud emocional y en la experiencia de bienestar de las víctimas de larga duración, destacando la soledad y el aislamiento social, lo cual podría relacionarse con una baja autoestima y síntomas depresivos. En cuanto a los agresores, podrían ser más propensos a involucrarse en una gran variedad de conductas desadaptativas y antisociales $\mathrm{y}$, al igual que las víctimas, tienen un mayor riesgo de depresión y de ideación suicida (Cowie, 2013). El informe de la Unesco (2018) sobre la violencia y el acoso escolar, realizado con estudiantes de entre 9 y 15 años de 144 países, indica que el bullying tiene un impacto significativo en la salud mental y física, la calidad de vida y el bienestar de las víctimas. Entre otras cosas, aquellos estudiantes que lo sufren tienen el doble de probabilidades de sentirse solos, y el triple de sentirse "extraños" en la escuela en relación con los que no son acosados y disminuye su sentido de pertenencia al centro educativo. En este sentido, sería interesante analizar la percepción del ambiente del aula en la etapa universitaria en relación con el acoso y la victimización por parte de los propios estudiantes.

El objetivo del presente trabajo es contribuir a analizar el bullying en una muestra universitaria española, concretamente estudiar cómo se distribuyen los diferentes roles implicados en el bullying (víctima, agresor, agresor victimizado y no implicado), estudiar la relación de dichos roles con la percepción que tienen los estudiantes del ambiente social del aula y su sensación de bienestar en ella, y comprobar las formas más frecuentes de agresión y de victimización en la muestra analizada.

\section{MÉtodo}

\subsection{Muestra}

El procedimiento de muestreo llevado a cabo es no probabilístico por conveniencia. La muestra está constituida por 776 estudiantes universitarios. Estudian cuatro titulaciones de grado (Magisterio en Educación Infantil, 
Magisterio en Educación Primaria, Educación Social y Psicología). El rango de edad varía entre los 17 y los 58 años, situándose el $95 \%$ de los estudiantes entre los 17 y los 26 años y siendo la media de 21,06 años y la DT de 3,775.

Del total de la muestra, 131 (16,9\%) son varones y $654(83,1 \%)$ son mujeres. La distribución por curso es la siguiente: 260 (33,5\%) de primer curso, $203(26,2 \%)$ de segundo curso, $137(17,7 \%)$ de tercer curso y $176(22,7 \%)$ de cuarto curso. Los participantes estudian cuatro titulaciones de grado (Magisterio en Educación Infantil, 279 (46 \%); Magisterio en Educación Primaria, 233 (30,1\%); Educación Social, 74 (9,4 \%), y Psicología, 190 (24,5\%).

\subsection{Variables e instrumentos}

Para evaluar la implicación en bullying de los estudiantes se ha utilizado la validación española desarrollada por Ortega-Ruiz, Del Rey y Casas (2016) del European Bullying Intervention Project Questionnaire (EBIP-Q). Este cuestionario ha sido elegido porque ha demostrado tener alta fiabilidad y por las ventajas y fortalezas de su validación (Ortega-Ruiz, Del Rey y Casas, 2016). Está formado por 14 ítems agrupados en dos dimensiones: victimización $(\Omega=0,86)$ y agresión $(\Omega=0,86$, evaluado a través del Omega de McDonald). Los ítems siguen una escala tipo Likert con cinco opciones de respuesta y se refieren a un intervalo temporal de los últimos dos meses: $0=$ no; 1 = sí, una o dos veces; 2 = sí, una o dos veces al mes; 3 = sí, alrededor de una vez a la semana; y 4 = sí, más de una vez a la semana. El cuestionario permite identificar los siguientes roles en bullying: víctima, agresor, agresor victimizado y no implicado.

Por otro lado, también se evaluó en cada estudiante su percepción del ambiente social del aula y su sensación de bienestar en ella, a través de cuatro ítems elaborados ad hoc con cuatro alternativas de respuesta (mucho, bastante, poco o nada). Concretamente los ítems son: "Me siento a gusto en esta clase"; "Considero que tengo amigos en clase"; "Puedo intervenir en clase sin problemas"; "Considero que hay buen ambiente en clase". La consistencia obtenida con los datos de este estudio mediante $\alpha$ de Cronbach fue de $\alpha=$ 0,799 . 


\subsection{Procedimiento}

Los instrumentos de evaluación se aplicaron en el primer cuatrimestre del curso 2017-18 a través de un enlace on line, de forma voluntaria y con la autorización paterna en el caso de los/as alumnos/as de primer curso menores de edad. Esta autorización se hizo llegar a los/as padres/madres en formato papel, donde se explicaba el objetivo del estudio y el proceso de gestión de datos. Al estudiante se explicó que las repuestas eran anónimas, confidenciales, que se requería sinceridad y que debían centrarse en lo ocurrido en el contexto universitario (no con los amigos de fuera de la Universidad) y durante los últimos dos meses.

\subsection{Análisis de datos}

Para calcular los roles de implicación en bullying en el cuestionario EBIP-Q se consideraron los criterios de frecuencia de participación y repetición, de acuerdo con los criterios de Del Rey et al. (2015, citado en Romera et al., 2017). Las víctimas fueron identificadas cuando obtuvieron puntuaciones mayores o iguales a 2 (una vez al mes) en cualquiera de los ítems de victimización y puntuaciones iguales o menores que 1 (una o dos veces) en todos los ítems de agresión. Los agresores fueron aquellos que marcaron 2 o más (una vez al mes) en algún ítem de agresión y 1 o 0 (una o dos veces o nunca) en todos los ítems de victimización. Los agresores victimizados puntuaron igual o mayor a 2 (una vez al mes) en cualquier ítem de la dimensión agresión y victimización. Los no implicados fueron considerados aquellos que obtuvieron una puntuación menor o igual que 1 (una o dos veces), tanto en los ítems de agresión como en los de victimización.

La clasificación de los participantes según los distintos roles de implicación en bullying se realizó a partir de los datos brutos a través de un programa escrito para R ( $R$ coreteam, 2018). Posteriormente, los datos fueron analizados con el paquete estadístico SPSS 24. Se calcularon los porcentajes de implicación en los diferentes roles del bullying y se analizaron las diferencias en la distribución de dichos roles en función del curso, del género y de la titulación, a través de la prueba de chi cuadrado $(\chi 2)$ y con la prueba z para la 
comparación de las proporciones. Por otro lado, se analizaron las diferencias de medias entre los roles del bullying en la percepción del ambiente social del aula y la sensación de bienestar en ella aplicando ANOVA factorial, con Bonferroni para los contrastes posteriores con un nivel de significación de 0,05 . Por último, se analizaron los porcentajes de las distintas formas de agresión y de victimización, para lo cual se agruparon las puntuaciones mayores o iguales a uno.

\subsection{Resultados}

Los resultados indican en la muestra total que el rol de víctima tiene un porcentaje de implicación del 12,4 \% (96 estudiantes), el rol de agresor del $5 \%$ (39 estudiantes) y el rol de agresor-victimizado, del 2,7 \% (21 estudiantes) (tabla 1).

La prueba de chi cuadrado muestra que la distribución de los roles en función del curso es estadísticamente diferente $(\chi 2=41,993, \mathrm{gl}=9$, $\operatorname{sig}=, 000)$. Centrándonos en las víctimas, se establecen dos grupos estadísticamente diferentes entre sí: los alumnos de $1 .^{\circ}$ y $2 .^{\circ}$ por un lado $(8,1$ y $7,9 \%$ respectivamente) y los estudiantes de $3 .^{\circ}$ y $4 .^{\circ}$ por otro lado (17,5 y $\left.19,9 \%\right)$, encontrando un mayor porcentaje de víctimas de bullying en los cursos superiores. Respecto a los agresores, los resultados indican que el porcentaje de implicación en el rol de agresor va aumentando desde el primer curso $(2,3 \%)$, pasando por segundo $(3,9 \%)$ y tercer cursos $(5,8 \%)$, hasta el cuarto $(9,7 \%)$, encontrando diferencias estadísticamente significativas entre $4 .^{\circ}$ y $1 .^{\circ}$. Por otro lado, la distribución del porcentaje de implicación en el rol de agresor victimizado es estadísticamente similar en todos los cursos, siendo este, además, el rol menos frecuente, como cabría esperar (tabla 1) 
TABLA 1

Distribución de los roles de bullying en función del curso y en la muestra total

\begin{tabular}{|c|c|c|c|c|c|c|c|}
\hline & & & \multicolumn{4}{|c|}{ Curso } & \multirow[t]{2}{*}{ Total } \\
\hline & & & $1^{o}$ & $2^{o}$ & $3^{\circ}$ & $4^{o}$ & \\
\hline \multirow{8}{*}{$\begin{array}{l}\text { Roles en } \\
\text { bullying }\end{array}$} & \multirow[b]{2}{*}{ No Implicado } & Recuento & 230 & $173_{\mathrm{a}}$ & $99_{b}$ & $118_{\mathrm{b}}$ & 620 \\
\hline & & $\begin{array}{l}\% \text { dentro de } \\
\text { curso }\end{array}$ & $88,5 \%$ & $85,2 \%$ & $72,3 \%$ & $67,0 \%$ & $79,9 \%$ \\
\hline & \multirow[b]{2}{*}{ Víctima } & Recuento & $21_{a}$ & $16_{a}$ & $24_{b}$ & $35_{b}$ & 96 \\
\hline & & $\begin{array}{l}\% \text { dentro de } \\
\text { curso }\end{array}$ & $8,1 \%$ & $7,9 \%$ & $17,5 \%$ & $19,9 \%$ & $12,4 \%$ \\
\hline & \multirow[b]{2}{*}{ Agresor } & Recuento & 6 & $8_{a, b}$ & $8_{a, b}$ & $17_{\mathrm{b}}$ & 39 \\
\hline & & $\begin{array}{l}\% \text { dentro de } \\
\text { curso }\end{array}$ & $2,3 \%$ & $3,9 \%$ & $5,8 \%$ & $9,7 \%$ & $5,0 \%$ \\
\hline & \multirow{2}{*}{$\begin{array}{l}\text { Agresor Victi- } \\
\text { mizado }\end{array}$} & Recuento & $3 \mathrm{a}$ & 6 a & 6 a & $6_{a}$ & 21 \\
\hline & & $\begin{array}{l}\% \text { dentro de } \\
\text { curso }\end{array}$ & $1,2 \%$ & $3,0 \%$ & $4,4 \%$ & $3,4 \%$ & $2,7 \%$ \\
\hline \multirow{2}{*}{\multicolumn{2}{|c|}{ Total }} & Recuento & 260 & 203 & 137 & 176 & 776 \\
\hline & & $\begin{array}{l}\% \text { dentro de } \\
\text { curso }\end{array}$ & $100,0 \%$ & $100,0 \%$ & $100,0 \%$ & $100,0 \%$ & $100,0 \%$ \\
\hline
\end{tabular}

Nota: Cada letra del subíndice denota un subconjunto de la categoría "Curso" cuyas proporciones de columna no difieren de forma significativa entre sí en el nivel ,05.

Fuente: Elaboración propia.

Respecto al género, los resultados indican que no existen diferencias significativas en la distribución de los roles del bullying entre hombres y mujeres $(\chi 2=1,514, \mathrm{gl}=3$, sig $=, 679)$, siendo los porcentajes de implicación de los roles similares entre las chicas y los chicos $(11,9$ y $14,5 \%$ respectivamente para el rol de víctima, el 5,1 y 4,6\% para el rol de agresor y el 2,5 y 3,8 \% para el de agresor victimizado). Somos conscientes de que nuestra muestra es desigual al tratarse de estudios universitarios en los que se matriculan más chicas que chicos, pero nos interesaba ver cómo se distribuyen los diferentes roles del bullying y si están presentes tanto en varones como en mujeres (tabla 2). 
TABLA 2

Distribución de los roles de bullying en función del género

\begin{tabular}{|c|c|c|c|c|c|}
\hline & & & \multicolumn{2}{|c|}{ Sexo } & Total \\
\hline & & & Mujer & Hombre & \\
\hline \multirow{8}{*}{ Roles en bullying } & \multirow{2}{*}{ No Implicado } & Recuento & $519 \mathrm{a}$ & $101_{\mathrm{a}}$ & 620 \\
\hline & & $\%$ dentro de sexo & $80,5 \%$ & $77,1 \%$ & $79,9 \%$ \\
\hline & \multirow{2}{*}{ Víctima } & Recuento & $77 \mathrm{a}$ & $19 \mathrm{a}$ & 96 \\
\hline & & $\%$ dentro de sexo & $11,9 \%$ & $14,5 \%$ & $12,4 \%$ \\
\hline & \multirow{2}{*}{ Agresor } & Recuento & $33_{\mathrm{a}}$ & $6_{a}$ & 39 \\
\hline & & $\%$ dentro de sexo & $5,1 \%$ & $4,6 \%$ & $5,0 \%$ \\
\hline & \multirow{2}{*}{ Agresor victimizado } & Recuento & $16_{a}$ & 5 a & 21 \\
\hline & & $\%$ dentro de sexo & $2,5 \%$ & $3,8 \%$ & $2,7 \%$ \\
\hline \multirow{2}{*}{\multicolumn{2}{|c|}{ Total }} & Recuento & 645 & 131 & 776 \\
\hline & & $\%$ dentro de sexo & $100,0 \%$ & $100,0 \%$ & $100,0 \%$ \\
\hline
\end{tabular}

Nota: Cada letra del subíndice denota un subconjunto de la categoría "Sexo" cuyas proporciones de columna no difieren de forma significativa entre sí en el nivel ,05.

Fuente: Elaboración propia.

Como se observa en las tablas 3 y 4 , el orden en que aparecen las conductas de victimización y de agresión en función del porcentaje de implicación es prácticamente el mismo en los dos grupos (de bullying menos frecuente y frecuente).

Los resultados indican que las formas más repetidas de victimización en los estudiantes universitarios de nuestra muestra (tanto en el grupo de bullying menos frecuente como más frecuente) son: ser insultado a través de terceras personas, ser excluido o ignorado, ser insultado directamente y ser el blanco de la difusión de rumores. Un porcentaje menor, pero también significativo de estudiantes señala que alguien le ha robado o roto sus cosas, que ha sido amenazado, o que ha sido golpeado, pateado o empujado (tabla 3 y figura 1). 
TABLA 3

Distribución de las formas de victimización en bullying

\begin{tabular}{|c|c|c|c|c|c|c|}
\hline \multirow[t]{2}{*}{ Formas de victimización en bullying } & \multicolumn{2}{|c|}{$\begin{array}{c}\text { Más de } 1 \text { o } 2 \\
\text { veces en los dos } \\
\text { últimos meses } \\
\text { (frecuente) }\end{array}$} & \multicolumn{2}{|c|}{$\begin{array}{l}1 \text { o } 2 \text { veces en } \\
\text { los dos últimos } \\
\text { meses (menos } \\
\text { frecuente) }\end{array}$} & \multicolumn{2}{|c|}{ Total } \\
\hline & $\%$ & $F$ & $\%$ & $F$ & $\%$ & $F$ \\
\hline $\begin{array}{l}\text { Alguien le ha dicho a otras personas palabras } \\
\text { malsonantes sobre mí (insultado) }\end{array}$ & $8,9 \%$ & 69 & $23,2 \%$ & 180 & $32,1 \%$ & 249 \\
\hline He sido excluido o ignorado por otras personas & $7,9 \%$ & 61 & $22,8 \%$ & 177 & $30,7 \%$ & 238 \\
\hline $\begin{array}{l}\text { Alguien me ha insultado y me ha dicho palabras } \\
\text { malsonantes }\end{array}$ & $4,8 \%$ & 37 & $17,9 \%$ & 139 & $22,7 \%$ & 176 \\
\hline Alguien ha difundido rumores sobre mí & $4,3 \%$ & 33 & $19,7 \%$ & 153 & $24 \%$ & 186 \\
\hline Alguien me ha robado o roto mis cosas & $0,8 \%$ & 6 & $6,6 \%$ & 51 & $7,4 \%$ & 57 \\
\hline Alguien me ha amenazado & $0,6 \%$ & 5 & $4,6 \%$ & 36 & $5,2 \%$ & 41 \\
\hline Alguien me ha golpeado, pateado o empujado & $0,5 \%$ & 4 & $5,5 \%$ & 27 & $6 \%$ & 31 \\
\hline
\end{tabular}

Fuente: Elaboración propia.

FIGURA 1

Formas de victimización en bullying

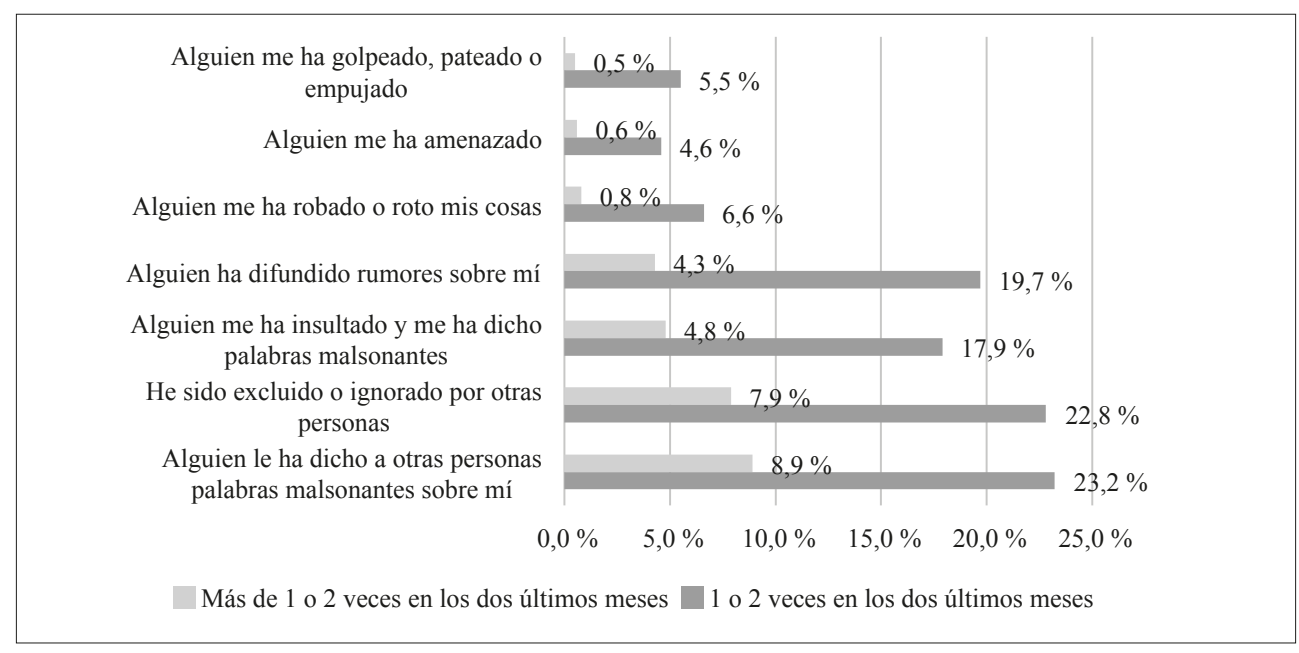

Fuente: Elaboración propia. 
A su vez, las formas más repetidas de agresión en los estudiantes universitarios de nuestra muestra son fundamentalmente decir palabras malsonantes (insultar) a través de terceras personas o directamente, excluir o ignorar a alguien y difundir rumores sobre alguien. Seguido a bastante distancia encontramos robar o estropear algo a alguien, golpear, patear o empujar a alguien y amenazar a alguien (tabla 4 y gráfico 2 ).

Los estudiantes universitarios que están implicados en bullying tienen una percepción diferente del clima de la clase y de su bienestar en ella. Así, el ANOVA muestra diferencias significativas en función de los roles de implicación en bullying en la percepción del ambiente social del aula en los cuatro ítems evaluados. Se observan diferencias significativas en todos los ítems considerados en este sentido (tabla 5).

Como se observa en la tabla 6 , al hacer los contrastes para ver específicamente entre qué roles de implicación existen diferencias encontramos que los más sensibles en este sentido son los estudiantes que tienen rol de víctima. Los resultados indican que estos: se sienten menos a gusto en clase que sus compañeros no implicados en situaciones bullying $(\mathrm{p}=0,00)$ y que los que tienen rol de agresor $(p=0,019)$; consideran que tienen menos amigos en clase que los no implicados $(p=0,00)$ y que los agresores $(p=0,023)$; tienen una peor percepción de la posibilidad de intervenir en clase sin problemas que los estudiantes no implicados $(\mathrm{p}=0,00)$; y consideran que hay peor ambiente en clase que los no implicados $(\mathrm{p}=0,00)$.

Por su parte, los estudiantes con el rol de agresor se sienten más a gusto en clase y consideran que tienen más amigos en ella que los estudiantes que son víctima de bullying ( $\mathrm{p}=0,019$ y $\mathrm{p}=0,023$, respectivamente). En comparación con los estudiantes no implicados, los agresores consideran que hay un peor ambiente general en clase $(p=0,010)$, pero ellos se sienten estadísticamente igual de a gusto en clase que los no implicados, creen que tienen amigos en clase en la misma medida, y piensan que pueden intervenir en clase sin problemas igual que los no implicados.

Los estudiantes con el rol de agresor-victimizado se sienten significativamente menos a gusto en clase $(\mathrm{p}=0,034)$ y piensan que hay peor ambiente en clase $(\mathrm{p}=0,002)$ que los estudiantes no implicados en bullying. 


\section{TABLA 4}

Distribución de las formas de agresión en bullying

\begin{tabular}{|c|c|c|c|c|c|c|}
\hline \multirow[t]{2}{*}{ Formas de agresión en bullying } & \multicolumn{2}{|c|}{$\begin{array}{c}\text { Más de } 1 \text { o } 2 \\
\text { veces en los dos } \\
\text { últimos meses } \\
\text { (frecuente) }\end{array}$} & \multicolumn{2}{|c|}{$\begin{array}{l}1 \text { o } 2 \text { veces en } \\
\text { los dos últimos } \\
\text { meses (menos } \\
\text { frecuente) }\end{array}$} & \multicolumn{2}{|c|}{ Total } \\
\hline & $\%$ & $F$ & $\%$ & $F$ & $\%$ & $F$ \\
\hline $\begin{array}{l}\text { He dicho a otras personas palabras malsonantes } \\
\text { sobre alguien }\end{array}$ & $5,7 \%$ & 44 & $31,1 \%$ & 241 & $36,8 \%$ & 285 \\
\hline $\begin{array}{l}\text { He insultado y he dicho palabras malsonantes } \\
\text { sobre alguien }\end{array}$ & $3,6 \%$ & 28 & $20,5 \%$ & 159 & $24,1 \%$ & 187 \\
\hline He excluido o ignorado a alguien & $1,9 \%$ & 15 & $16,1 \%$ & 125 & $18 \%$ & 140 \\
\hline He difundido rumores sobre alguien & $0,5 \%$ & 4 & $7,3 \%$ & 57 & $7,8 \%$ & 61 \\
\hline He robado o he estropeado algo a alguien & $0,1 \%$ & 1 & $1,7 \%$ & 13 & $1,8 \%$ & 14 \\
\hline He golpeado, pateado o empujado a alguien & $0,0 \%$ & 0 & $1,5 \%$ & 12 & $1,5 \%$ & 12 \\
\hline He amenazado a alguien & $0,0 \%$ & 0 & $0,8 \%$ & 6 & $0,8 \%$ & 6 \\
\hline
\end{tabular}

Fuente: Elaboración propia.

\section{FIGURA 2}

Formas de agresión en bullying

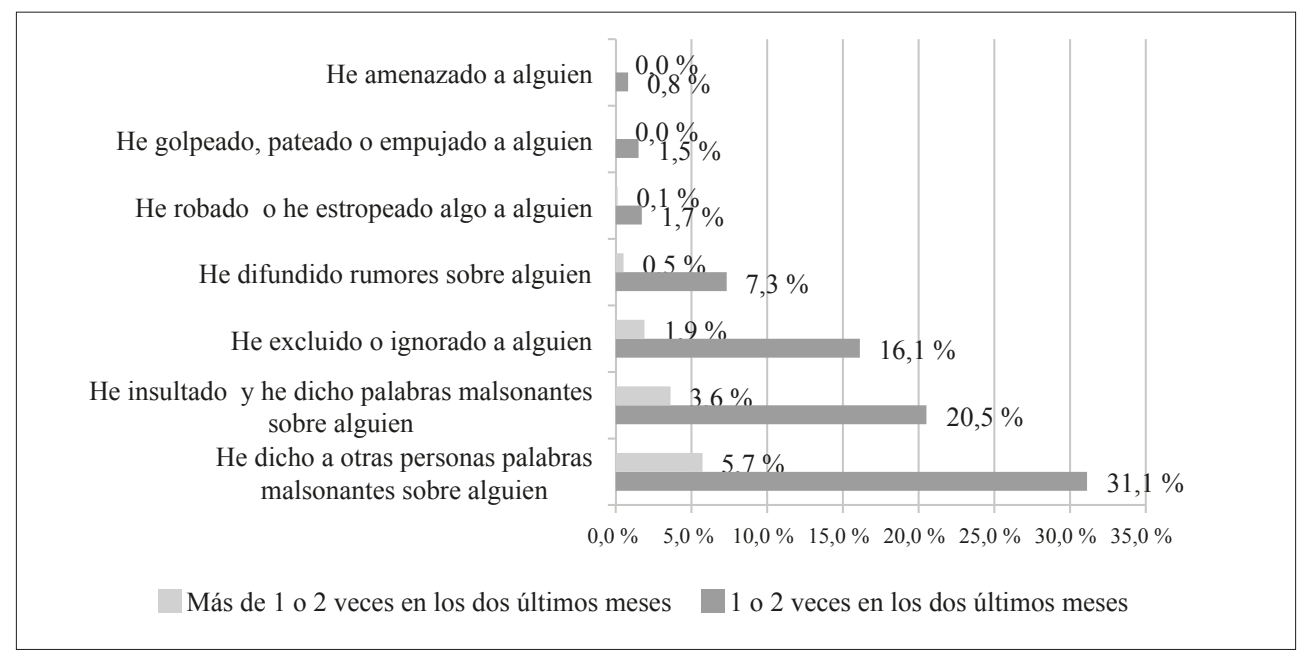

Fuente: Elaboración propia. 
TABLA 5

Media, desviación típica, F y Significación del ANOVA en la percepción del ambiente social del aula en función de los roles de implicación en bullying

\begin{tabular}{|c|c|c|c|c|c|c|}
\hline & & $N$ & Media & $D T$ & $F$ & Sig \\
\hline \multirow{5}{*}{$\begin{array}{l}\text { Me siento a gusto en esta } \\
\text { clase }\end{array}$} & No implicado & 620 & 2,40 & 0,761 & 21,244 & 0,000 \\
\hline & Víctima & 96 & 1,73 & 1,000 & & \\
\hline & Agresor & 39 & 2,18 & 0,914 & & \\
\hline & Agresor victimizado & 21 & 1,90 & 0,700 & & \\
\hline & Total & 776 & 2,29 & 0,831 & & \\
\hline \multirow{5}{*}{$\begin{array}{l}\text { Considero que tengo amigos } \\
\text { en clase }\end{array}$} & No implicado & 620 & 1,52 & 0,637 & 6,599 & 0,000 \\
\hline & Víctima & 96 & 1,21 & 0,679 & & \\
\hline & Agresor & 39 & 1,56 & 0,680 & & \\
\hline & Agresor victimizado & 21 & 1,48 & 0,680 & & \\
\hline & Total & 776 & 1,48 & 0,652 & & \\
\hline \multirow{5}{*}{$\begin{array}{l}\text { Puedo intervenir en clase sin } \\
\text { problemas }\end{array}$} & No implicado & 620 & 2,41 & 0,756 & 6,763 & 0,000 \\
\hline & Víctima & 96 & 2,03 & 0,923 & & \\
\hline & Agresor & 39 & 2,36 & 0,843 & & \\
\hline & Agresor victimizado & 21 & 2,19 & 0,928 & & \\
\hline & Total & 776 & 2,36 & 0,797 & & \\
\hline \multirow{5}{*}{$\begin{array}{l}\text { Considero que hay buen } \\
\text { ambiente en clase }\end{array}$} & No implicado & 620 & 1,97 & 0,897 & 12,179 & 0,000 \\
\hline & Víctima & 96 & 1,53 & 1,025 & & \\
\hline & Agresor & 39 & 1,49 & 0,942 & & \\
\hline & Agresor victimizado & 21 & 1,24 & 0,995 & & \\
\hline & Total & 776 & 1,87 & 0,938 & & \\
\hline
\end{tabular}

Fuente: Elaboración propia. 
TABLA 6

Comparaciones múltiples entre los roles del bullying en la percepción del ambiente social del aula

\begin{tabular}{|c|c|c|c|c|c|c|c|}
\hline \multirow{2}{*}{$\begin{array}{c}\text { Variable } \\
\text { dependiente }\end{array}$} & \multirow{2}{*}{$\begin{array}{l}\text { (I) Roles en } \\
\text { bullying }\end{array}$} & \multirow{2}{*}{$\begin{array}{l}(J) \text { Roles en } \\
\text { bullying }\end{array}$} & \multirow{2}{*}{$\begin{array}{c}\text { Diferencia } \\
\text { de medias } \\
\quad(I-J)\end{array}$} & \multirow{2}{*}{$\begin{array}{l}\text { Error } \\
\text { típico }\end{array}$} & \multirow{2}{*}{ Sig. } & \multicolumn{2}{|c|}{$\begin{array}{c}\text { Intervalo de confianza } \\
\text { al } 95 \%\end{array}$} \\
\hline & & & & & & $\begin{array}{c}\text { Limite } \\
\text { superior }\end{array}$ & $\begin{array}{l}\text { Limite } \\
\text { inferior }\end{array}$ \\
\hline \multirow{12}{*}{$\begin{array}{l}\text { Me siento a } \\
\text { gusto en esta } \\
\text { clase }\end{array}$} & \multirow{3}{*}{ No implicado } & Víctima &, $668(*)$ & 0,088 & 0,000 & 0,44 & 0,90 \\
\hline & & Agresor & 0,217 & 0,132 & 0,604 & $-0,13$ & 0,57 \\
\hline & & $\begin{array}{l}\text { Agresor } \\
\text { victimizado }\end{array}$ &, $492(*)$ & 0,178 & 0,034 & 0,02 & 0,96 \\
\hline & \multirow{3}{*}{ Víctima } & No implicado &,$- 668(*)$ & 0,088 & 0,000 & $-0,90$ & $-0,44$ \\
\hline & & Agresor &,$- 450\left(^{*}\right)$ & 0,152 & 0,019 & $-0,85$ & $-0,05$ \\
\hline & & $\begin{array}{l}\text { Agresor } \\
\text { victimizado }\end{array}$ & $-0,176$ & 0,193 & 1,000 & $-0,69$ & 0,33 \\
\hline & \multirow{3}{*}{ Agresor } & No implicado & $-0,217$ & 0,132 & 0,604 & $-0,57$ & 0,13 \\
\hline & & Víctima &, $450(*)$ & 0,152 & 0,019 & 0,05 & 0,85 \\
\hline & & $\begin{array}{l}\text { Agresor } \\
\text { victimizado }\end{array}$ & 0,275 & 0,217 & 1,000 & $-0,30$ & 0,85 \\
\hline & \multirow{3}{*}{$\begin{array}{l}\text { Agresor victi- } \\
\text { mizado }\end{array}$} & No implicado &,$- 492\left(^{*}\right)$ & 0,178 & 0,034 & $-0,96$ & $-0,02$ \\
\hline & & Víctima & 0,176 & 0,193 & 1,000 & $-0,33$ & 0,69 \\
\hline & & Agresor & $-0,275$ & 0,217 & 1,000 & $-0,85$ & 0,30 \\
\hline \multirow{12}{*}{$\begin{array}{l}\text { Considero que } \\
\text { tengo amigos } \\
\text { en clase }\end{array}$} & \multirow{3}{*}{ No implicado } & Víctima &, $309\left(^{*}\right)$ & 0,071 & 0,000 & 0,12 & 0,50 \\
\hline & & Agresor & $-0,046$ & 0,107 & 1,000 & $-0,33$ & 0,24 \\
\hline & & $\begin{array}{l}\text { Agresor } \\
\text { victimizado }\end{array}$ & 0,042 & 0,143 & 1,000 & $-0,34$ & 0,42 \\
\hline & \multirow{3}{*}{ Víctima } & No implicado &,$- 309\left(^{*}\right)$ & 0,071 & 0,000 & $-0,50$ & $-0,12$ \\
\hline & & Agresor &,$- 356\left(^{*}\right)$ & 0,123 & 0,023 & $-0,68$ & $-0,03$ \\
\hline & & $\begin{array}{l}\text { Agresor } \\
\text { victimizado }\end{array}$ & $-0,268$ & 0,155 & 0,512 & $-0,68$ & 0,14 \\
\hline & \multirow{3}{*}{ Agresor } & No implicado & 0,046 & 0,107 & 1,000 & $-0,24$ & 0,33 \\
\hline & & Víctima &, $356\left(^{*}\right)$ & 0,123 & 0,023 & 0,03 & 0,68 \\
\hline & & $\begin{array}{l}\text { Agresor } \\
\text { victimizado }\end{array}$ & 0,088 & 0,175 & 1,000 & $-0,37$ & 0,55 \\
\hline & \multirow{3}{*}{$\begin{array}{l}\text { Agresor victi- } \\
\text { mizado }\end{array}$} & No implicado & $-0,042$ & 0,143 & 1,000 & $-0,42$ & 0,34 \\
\hline & & Víctima & 0,268 & 0,155 & 0,512 & $-0,14$ & 0,68 \\
\hline & & Agresor & $-0,088$ & 0,175 & 1,000 & $-0,55$ & 0,37 \\
\hline
\end{tabular}




\begin{tabular}{|c|c|c|c|c|c|c|c|}
\hline \multirow{2}{*}{$\begin{array}{c}\text { Variable } \\
\text { dependiente }\end{array}$} & \multirow{2}{*}{$\begin{array}{l}\text { (I) Roles en } \\
\text { bullying }\end{array}$} & \multirow{2}{*}{$\begin{array}{l}\text { (J) Roles en } \\
\text { bullying }\end{array}$} & \multirow{2}{*}{$\begin{array}{c}\text { Diferencia } \\
\text { de medias } \\
\quad(I-J)\end{array}$} & \multirow{2}{*}{$\begin{array}{l}\text { Error } \\
\text { típico }\end{array}$} & \multirow{2}{*}{ Sig. } & \multicolumn{2}{|c|}{$\begin{array}{c}\text { Intervalo de confianza } \\
\text { al } 95 \%\end{array}$} \\
\hline & & & & & & $\begin{array}{l}\text { Limite } \\
\text { superior }\end{array}$ & $\begin{array}{l}\text { Limite } \\
\text { inferior }\end{array}$ \\
\hline \multirow{12}{*}{$\begin{array}{l}\text { Puedo inter- } \\
\text { venir en clase } \\
\text { sin problemas }\end{array}$} & \multirow{3}{*}{ No implicado } & Víctima &, $380\left(^{*}\right)$ & 0,086 & 0,000 & 0,15 & 0,61 \\
\hline & & Agresor & 0,052 & 0,130 & 1,000 & $-0,29$ & 0,40 \\
\hline & & $\begin{array}{l}\text { Agresor } \\
\text { victimizado }\end{array}$ & 0,221 & 0,175 & 1,000 & $-0,24$ & 0,68 \\
\hline & \multirow{3}{*}{ Víctima } & No implicado &,$- 380(*)$ & 0,086 & 0,000 & $-0,61$ & $-0,15$ \\
\hline & & Agresor & $-0,328$ & 0,150 & 0,173 & $-0,72$ & 0,07 \\
\hline & & $\begin{array}{l}\text { Agresor } \\
\text { victimizado }\end{array}$ & $-0,159$ & 0,190 & 1,000 & $-0,66$ & 0,34 \\
\hline & \multirow{3}{*}{ Agresor } & No implicado & $-0,052$ & 0,130 & 1,000 & $-0,40$ & 0,29 \\
\hline & & Víctima & 0,328 & 0,150 & 0,173 & $-0,07$ & 0,72 \\
\hline & & $\begin{array}{l}\text { Agresor } \\
\text { victimizado }\end{array}$ & 0,168 & 0,213 & 1,000 & $-0,40$ & 0,73 \\
\hline & \multirow{3}{*}{$\begin{array}{l}\text { Agresor victi- } \\
\text { mizado }\end{array}$} & No implicado & $-0,221$ & 0,175 & 1,000 & $-0,68$ & 0,24 \\
\hline & & Víctima & 0,159 & 0,190 & 1,000 & $-0,34$ & 0,66 \\
\hline & & Agresor & $-0,168$ & 0,213 & 1,000 & $-0,73$ & 0,40 \\
\hline \multirow{12}{*}{$\begin{array}{l}\text { Considero } \\
\text { que hay buen } \\
\text { ambiente en } \\
\text { clase }\end{array}$} & \multirow{3}{*}{ No implicado } & Víctima &, $435(*)$ & 0,101 & 0,000 & 0,17 & 0,70 \\
\hline & & Agresor &, $479(*)$ & 0,152 & 0,010 & 0,08 & 0,88 \\
\hline & & $\begin{array}{l}\text { Agresor } \\
\text { victimizado }\end{array}$ &, $728(*)$ & 0,204 & 0,002 & 0,19 & 1,27 \\
\hline & \multirow{3}{*}{ Víctima } & No implicado &,$- 435\left(^{*}\right)$ & 0,101 & 0,000 & $-0,70$ & $-0,17$ \\
\hline & & Agresor & 0,044 & 0,174 & 1,000 & $-0,42$ & 0,51 \\
\hline & & $\begin{array}{l}\text { Agresor } \\
\text { victimizado }\end{array}$ & 0,293 & 0,221 & 1,000 & $-0,29$ & 0,88 \\
\hline & \multirow{3}{*}{ Agresor } & No implicado &,$- 479(*)$ & 0,152 & 0,010 & $-0,88$ & $-0,08$ \\
\hline & & Víctima & $-0,044$ & 0,174 & 1,000 & $-0,51$ & 0,42 \\
\hline & & $\begin{array}{l}\text { Agresor } \\
\text { victimizado }\end{array}$ & 0,249 & 0,249 & 1,000 & $-0,41$ & 0,91 \\
\hline & \multirow{3}{*}{$\begin{array}{l}\text { Agresor victi- } \\
\text { mizado }\end{array}$} & No implicado &,$- 728(*)$ & 0,204 & 0,002 & $-1,27$ & $-0,19$ \\
\hline & & Víctima & $-0,293$ & 0,221 & 1,000 & $-0,88$ & 0,29 \\
\hline & & Agresor & $-0,249$ & 0,249 & 1,000 & $-0,91$ & 0,41 \\
\hline
\end{tabular}

Fuente: Elaboración propia. 


\section{Discusiones y RESUltados}

Resulta paradójico pensar que pueda producirse acoso en personas con mayor formación y algunos autores sugieren una mayor brutalidad de las acciones con mayores efectos sobre las víctimas (Prieto, Carrillo y Lucio, 2015). Con el presente estudio, se pone de manifiesto que se producen situaciones de acoso en los centros universitarios españoles evaluados, en una frecuencia que consideramos suficientemente relevante como para ser tenida en cuenta. El 12,4 \% de los participantes expresaron haber sufrido acoso en la Universidad, una frecuencia que se suma a la diversidad transnacional encontrada en diferentes estudios (Pörhörla et al., 2016; Hoyos, Llanos y Valega, 2012; Paredes, Sanabria-Ferrand, González-Quevedo y Moreno, 2010). Nos encontramos por encima de las frecuencias encontradas en Finlandia, Reino Unido o Estonia, por debajo de muestras de Argentina o similares a los encontrados por Hoyos, Llanos y Valega (2012) con población colombiana. En cierta manera, podríamos estar aportando un dato explicable por la conceptualización del acoso en cada país, o bien tratando con datos incomparables dada la diversidad en la definición y/o procedimiento de evaluación del término estudiado.

Si nos fijamos en los datos recientes con población infanto-juvenil en España, que sitúa el acoso escolar en un 9,3\% (Sastre, 2016), no se ha detectado una disminución de acoso universitario en la muestra estudiada (situado en un $12,4 \%$ ), como era de esperar a partir de la investigación clásica en este tema (Olweus, 1998).

En cuanto a los agresores, el $5 \%$ detectado en la muestra española del presente artículo se sitúa entre las frecuencias del 1,7\% del estudio con universitarios finlandeses de Kunttu y Huttunen (2009) y el $12 \%$ del estudio con universitarios colombianos de Hoyos, López y Valega (2012).

Un hecho especialmente llamativo es que las frecuencias tanto en el rol de víctimas como en el de agresor aumentan significativamente en los últimos cursos. Cabría esperar que la mayor madurez de los estudiantes del último curso, las mayores competencias académicas adquiridas y sus expectativas profesionales más cercanas los llevaran a tener relaciones interpersonales más adecuadas y más habilidades para una convivencia positiva, pero es el curso en el que más casos encontramos tanto de víctimas (19,9\%) como de agresores $(9,7 \%)$, lo que podría deberse, entre otras cosas, a la mayor tensión 
acumulada en este último curso o a que la Universidad no está cumpliendo su objetivo de favorecer el desarrollo de competencias transversales como, por ejemplo, la resolución de problemas, el trabajo en equipo o las habilidades sociales.

En cuanto al sexo, no se han encontrado diferencias significativas en ninguno de los roles, frente a los estudios que confirman que los hombres son más veces agresores que las mujeres (Pörhöblä, Cvancara, Kaal, Tampere y Torres, 2016; Hoyos, Llanos y Valega, 2012) o que las mujeres se relacionan con mayor frecuencia con el rol de testigo (Hoyos, Llanos y Valega, 2012). Respecto al rol de víctima, los estudios han aportado datos contradictorios.

En cuanto al tipo de conductas recibidas por las víctimas, teniendo en cuenta el grupo total, las más frecuentes han sido "ser insultado a través de terceras personas $(32,1 \%)$, "ser excluido o ignorado" (30,7\%) y "difundir rumores sobre él" (24\%), resultados similares a los encontrados en otras investigaciones, donde además se constata que las agresiones de tipo físico (4\% en el presente estudio) son las menos frecuentes en poblaciones universitarias (Vergel, Martínez y Zafra, 2016; Cowie y Myers, 2015; Hoyos, Llanos y Valega, 2012).

Atendiendo a los tipos de agresión, las más frecuentes han sido "decir palabras malsonantes sobre otros o directamente" (36,7 \%), "excluir o ignorar" $(24,1 \%)$ y "difundir rumores" $(7,9 \%)$, formas de acoso similares a las encontradas en otros estudios (Pottinger y Stair, 2009).

Los resultados respecto a las conductas del grupo de bullying considerado frecuente (más de 1 o 2 veces en los dos últimos meses) resultan coherentes con los porcentajes de prevalencia del acoso encontrados en este estudio (el $12,4 \%$ para el rol de víctima y el $5 \%$ para el rol de agresor), puesto que se sitúan en torno a dicha cifra, descendiendo en las formas más extremas de violencia.

En el grupo de bullying menos frecuente y en el grupo total, las conductas agresivas con mayor porcentaje implican normalización de unas conductas que los estudiantes no parecen identificar con agresión (insultar o decir palabras malsonantes), y es posible que sepan diferenciar cuándo tienen intencionalidad negativa y cuándo no. Pero las otras conductas (como "robar o estropear", 1,8 \%; "maltrato físico", 1,5\% o "amenazar", 0,8 \%), a pesar de su menor frecuencia, consideramos que son lo suficientemente graves como 
para diseñar estrategias específicas de intervención y prevención. Debemos recordar que son los propios estudiantes quienes se identifican a sí mismos como agentes de la agresión, es decir, ellos indican que han robado o estropeado, excluido o ignorado, o difundido rumores sobre otros. Sería necesario analizar la percepción del agresor sobre estas conductas, pues cabría la opción de que no fueran conscientes de que están cometiendo un tipo de abuso.

Aunque la investigación realizada no explora las consecuencias psicológicas de los roles asociados al acoso, se disponen de algunos datos sobre bienestar en el aula. Así, las víctimas se sienten menos a gusto en clase y expresan tener menos amigos que los no implicados o los agresores; además, sienten que pueden intervenir menos en clase y perciben un peor ambiente que los no implicados. Sería interesante/necesario analizar si esta vivencia de la experiencia de aula repercute en alguna otra variable relacionada con el bienestar psicológico, el rendimiento académico o las relaciones sociales tal y como han demostrado diferentes estudios (Isaacs et al., 2008), teniendo en cuenta su relevancia dado que las titulaciones estudiadas en este trabajo están orientadas a profesiones muy vinculadas a la educación y al desarrollo de las personas. Sentirse bien en clase es importante, ya que se ha visto que dos de las principales condiciones de protección contra el acoso, son tener una buena integración en el grupo de iguales en clase y tener buenos amigos (Díaz-Aguado, Martínez Arias y Martín Babarro, 2010).

Principalmente en el caso de las titulaciones de Magisterio, consideramos que la experiencia de aula y de relaciones con los compañeros serán aspectos fundamentales para su desempeño profesional futuro. En cualquier caso, nuestros resultados sugieren que los alumnos que se identifican como víctimas son conscientes de su situación y no lo están pasando bien, lo que indica la necesidad de realizar actuaciones de prevención, detección e intervención en bullying también en contextos universitarios.

En definitiva, los datos aportados nos indican que el acoso existe en la Universidad. Si como apuntan algunas investigaciones este hecho podría estar condicionado por las propias características de la Universidad (Vergel, Martínez y Zafra, 2016; McDougall, citado en Hoyos, Llanos y Velga, 2012), como las relacionadas con modelos de gestión, liderazgo, sistemas de evaluación, etc. (Coleyshaw, 2010), será necesario focalizar la investigación en 
la identificación de estos factores con el objetivo de poder encontrar nuevos modelos que favorezcan unas relaciones más positivas.

\section{BiBLIOGRAFÍA}

Adams, F. D. y Lawrence, G. J. (2011). Bullying victims: The effects last into college. American Secondary Education 40(1), 4-11.

Benites, M. (2012). La convivencia escolar: una estrategia de intervención en bullying, en L. Benites, J. Carozzo, V. Horna, L. Palomino, C. Salgado, C. Uribe y L. Zapata (eds.), Bullying y convivencia en la escuela. Aspectos conceptuales (pp. 75-104). Lima: Observatorio sobre Violencia y Convivencia en la Escuela.

Caravaca, F., Falcón M., Navarro-Zaragoza, J., Luna Ruiz-Cabello, A., Rodriges, O. y Luna Maldonado, A (2012). Prevalence and patterns of traditional bullying victimization and cyber-teasing among college population in Spain. Revista BMC public health, vol. 16, 176. DOI: <https:// doi.org/10.1186/s12889-016-2857-8>.

Chapell, M. S., Hasselman, S. L., Kitchin, T., Lomon, S. N., MacIver, K. W. y Sarullo, P. L. (2006). Bullying in Elementary School, High School and College. Adolescence 41(164), 633-648.

Coleyshaw, L. (2010). The power of paradigms: a discussion of the abscence of bullying research in the context of the university student experience. Research in Post-Compulsory Education (4), 377-386. DOI: <https://doi. org/10.1080/13596748.2010.526799>.

CowIE, H. A. (2013) Cyberbullying and its impact on young people's emotional health and well-being. The Psychiatrist 37, 167-170. DOI: $<$ https:// doi.org/10.1192/pb.bp.112.040840>.

Cowie, H. y Myers, C. A. (2014). Bullying amongst University Students in the UK. International Journal of Emotional Education 6(1), 66-75.

Cowie, H. y Myers, C. A. (2015). Bullying Among University Students: Cross-national Perspectives. Abingdon: Routhledge. DOI: <https://doi. org/10.4324/9781315750132>. 
Curwen, T., McNicho, J. S. y Sharpe, G. (2011). The Progression of Bullying from Elementary School to University. International Journal of Humanities and Social Science 1(13), 47-54.

Díaz-Aguado, M. J., Martínez-Arias, R. y Martín Babarro, J. (2010). Estudio estatal de la convivencia escolar en la Educación Secundaria Obligatoria. Madrid: Ministerio de Educación.

DoĞRuer, N. y Yaratan, H. (2014). Developing a Bullying Scale for Use with University Students. Social Behavior and Personality: an International Journal 42(1), 81S-92S. DOI: <https://doi.org/10.2224/ sbp.2014.42.0.S81>.

García Peña, J. J., Moncada Ortíz, R. M. y Quintero Gil, J. (2013). El bullying y el suicidio en el escenario universitario. Revista Colombiana de Ciencias Sociales 4(2), 298-310. DOI: <https://doi.org/10.21501/ issn.2216-1201>.

GoldSMID, S. y Howie, P. (2014). Bullying by definition: an examination of definitional components of bullying. Emotional and Behavioural Difficulties 19(2), 210-225. DOI: <https://doi.org/10.1080/13632752.2013.84441 $4>$.

Hoyos, O., Romero, L., Valega, S. J. y Molinero, C. (2009). El maltrato entre iguales por abuso de poder exclusión social y en estudiantes de una universidad privada de la ciudad de Barranquillas. Revista Pensamiento Psicológico 6(13), 109-126.

Hoyos, O. L. R., Llanos M. M. y Valega, S. J. (2012). El maltrato entre iguales por abuso de poder en el contexto universitario: incidencia, manifestaciones y estrategias de solución. Universitas Psychologica 11(3), 793-802. Isaacs, J., Hodges, E. y Salmivalli, C. (2008). Long-term consequences of victimization: A follow-up from adolescence to young adulthood. European Journal of Developmental Science 2, 387-397.

Kunttu, K. y Huttunen, T. (2009). Student health survey 2008: A national survey among Finnish university students. Helsinki: Finnish Student Health Service. Recuperado de: <http:/www.yths.fi/filebank/582-Tutkimus_2008_verkkoon.pdf $>$.

Lappalainen, C., Meriläinen, M., Puhakka, H. y Sinkkonen, H-M. (2011). Bullying among university students does it exist? Finnish Journal of Youth Research 29(2), 64-80. 
Meriläinen, M., Puhakka, H. y Sinkkonen, H.- M. (2015). Students suggestions for eliminating bullying at a university. British Journal of Guidance \& Counselling 43(2), 202-215. DOI: <https://doi.org/10.1080/03069885. 2014.950943>.

Monks, C. P., Smith, P. K., Naylor, P., Barter C, Ireland, J. L. y Coyne, I. (2009). Bullying in different contexts: Commonalities, differences and the role of theory. Aggression and Violent Behavior 14(2), 146-156. DOI: $<$ https://doi.org/10.1016/j.avb.2009.01.004>.

Nansel, T., Overpeck, M., Pilla, R., Ruan, W., Simon-Mortton, B. y Scheidt, P. (2001). Bullying behaviors among U.S. youth: Prevalence and association with psychosocial adjustment. Journal of the American Medical Association 285, 2094-2100. DOI: <https://doi.org/10.1001/jama.285.16.2094>.

NUS StUdEnt EXPERIENCE Report (2008). Retrieved February 17, 2012. Recuperado de: $<$ http://www.nus.org.uk/PageFiles/4017/NUS_StudentExperienceReport.pdf $>$.

Olweus, D. (1998). Conductas de acoso y amenazas entre escolares. Madrid: Morata.

Ortega, R., Del Rey, R. y CAsas, J. A. (2013). La convivencia escolar: clave en la predicción del Bullying. Revista Iberoamericana de Evaluación Educativa 6(2), 91-102. DOI: <https://doi.org/10.15366/riee>.

Paredes, O., Sanabria-Ferrand, P. A., González-Quevedo, L. A. y Moreno Rehalpe, S.P. (2010). "Bullying" en las facultades de medicina colombianas, mito o realidad. Revista Médica 18(2), 161-172.

Perry, A. y Blincoe, S. (2015). Bullies and Victims in Higher Education. Journal of Bullying and Social Aggression 1(1). Recuperado de: $<\mathrm{http}: / /$ sites. tamuc.edu/bullyingjournal/bullies-and-victims-in-higher-education/>.

Prieto Quezada, M. T., Carrillo Navarro, J. C. y Lucio López, L. A. (2015). Violencia virtual y acoso escolar entre estudiantes universitarios: el lado oscuro de las redes sociales. Innovación Educativa 15(68), 33-47.

Pörhölä, M., Cvancara, K., Kaal, E., Tampere, K. y Torres, B. (2016). Cross-cultural comparisons of bullying among university students: perspectives from Argentina, Estonia, Finland and the United States, en $\mathrm{H}$. Cowie y C.-A. Myers (eds.), Bullying among university students: cross-national perspectives (pp. 127-142). Abingdon: Routledge. 
Pottinger, A. y Stair, A. (2009). Bullying of students by teachers and peers and its effect on the psychological well-being of students in Jamaican schools. Journal of School Violence 8(4), 312-327.

Romera, E. M., Ortega, R., Del Rey, R., Casas, J. A. y cols. (2017) Bullying, cyberbullying y dating violence. Estudio de la gestión de la vida social en estudiantes de Primaria y Secundaria de Andalucía. Centro de Estudios Andaluces, Junta de Andalucía.

Romero, A. y Plata, J. V. (2015). Acoso Escolar en las Universidades. Revista Enseñanza e Investigación en Psicología 20(3), 266-274.

Salmivalli, C. (2010). Bullying and the peer-group: A review. Agression and Violent Behavior, 15(2), 112-120. DOI: <https://dx.doi.org/10.1016 j.avb.2009.08.007>.

Sastre, A. (coord.) (2016) Yo a eso no juego. Bullying y Ciberbullying en la infancia. Save the Children.

Sinkkonen, H. M., Puhakka, H. y MeniläInen, M. (2012). Bullying at a university: students' experiences of bullying. Studies in Higher Education 39(1), 1-13. DOI: <http://dx.doi.org/10.1080/03075079.2011.649726>.

Trujillo, J. J. y Romero-Acosta, K. (2016). Variables que evidencian el bullying en un contexto universitario. Revista Encuentros, Universidad Autónoma del Caribe, 14 (01), 41- 54. DOI: <http://dx.doi.org/10.15665/ re.v14i1.668>.

UNESCO (2018) School violence and bullying: Global status and trends, drivers and consequences. Recuperado de <http:/www.infocoponline.es/pdf/ BULLYING.pdf $>$.

Vásquez de la Hoz, F., Ávila Lugo, N., Márquez Chaparro, L., Martínez González, G., Mercado Espinosa, J. y Severiche Jiménez, J. (2010). Inteligencia emocional e índices de bullying en estudiantes de psicología de una universidad privada de Barranquilla, Colombia. Psicogente 13(24), 306-328.

Vergel, M., Martínez, J. y Zafra, S. (2016). Factores asociados al bullying en instituciones de educación superior. Revista Criminalidad 58(2), 197208. 
